\title{
Research Digest
}

\section{Synopses of Research Articles}

\author{
Nicotine Keeps Leaf-Loving \\ Herbivores at Bay \\ DOI: 10.1371/journal.pbio.0020250
}

Sooner or later, a gardener looking for "nontoxic" ways to control the inevitable attack on a favorite plant will discover the nicotine remedy. Steep a cup of loose tobacco in a gallon of water, let it sit overnight, strain, and spray away. Caterpillars, aphids, and a diverse array of insects predisposed to devouring plants will soon abandon your vegetables and flowers in search of less disagreeable forage.

The ultimate sitting duck, plants rely on an arsenal of chemical metabolites to fend off predators. Many of these chemicals harbor anti-herbivore properties, which have been exploited for commercial use. Nicotine, it turns out, is so toxic that it was one of the first chemicals used in agricultural insecticides. It's not clear, though, whether these toxic metabolites are really defending plants against hungry herbivores in their natural environment, especially since many insects can tolerate various plant chemicals and sometimes even incorporate them into their own defenses. Though scientists have cataloged a long list of these presumed resistance traits, there's no evidence that they offer plants a competitive advantage against their leaf-covetous foes in nature.

With plant and plant-eater engaged in an ever-escalating battle of evolutionary one-upmanship and with plants capable of producing an array of defensive responses, teasing out the predatorresistant effects of individual plant metabolites has proved challenging. Theoretically, one could track down a resistance gene by breeding plants that are genetically identical save for the gene that controls expression of a particular resistance trait. In practice, however, traditional breeding techniques aren't that precise and tend to generate additional variations in genomic regions that are linked to the target gene and that might affect resistance as well.

The tools of genetic engineering have largely overcome such limitations, allowing scientists far greater control and specificity. Following this approach, lan Baldwin and colleagues use transgenic silencing (which introduces gene "constructs" into an organism to inactivate a gene of interest) to investigate a single resistance trait, nicotine production. Even though nicotine is one of the best-studied putative resistance traits, its specific role has been unclear.

To isolate the resistance effects of nicotine from possible confounding factors, Baldwin and colleagues blocked with nonmutant cultivated plants, in southwestern Utah, N. attenuata's native habitat. A subset of the plants was also treated with a chemical known to increase both nicotine content and resistance to herbivore attack. Predictably, several of the plant's natural insect enemies made an appearance. Untreated nicotine-deficient transgenic plants fared the worst, losing twice as much foliage to herbivores as nonmutant plants. Transgenic plants treated with the chemical boost performed much better, showing about the same amount of damage as the nonmutants. Interestingly, tobacco hornworms-which, as their name implies, feed primarily on tobacco-preferred nicotine-free plants when given the

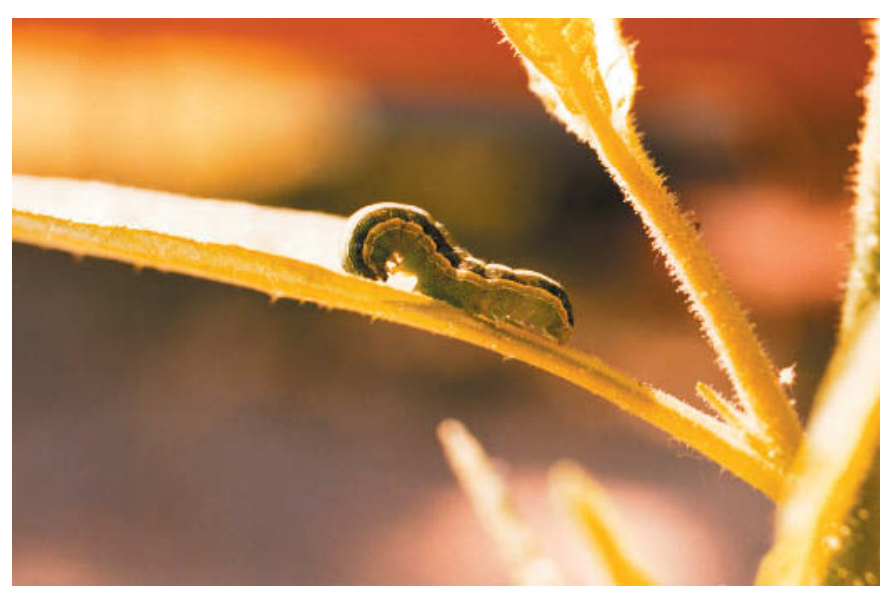

DOI: 10.1371/journal.pbio.0020250.g001

Spodoptera exigua larva feeding on Nicotiana attenuata choice. Though the worms have evolved strategies for coping with nicotine's deleterious effects, these adaptations come at a price: worms feeding on nicotine-deficient tobacco grew bigger and faster than those feeding on plants with normal nicotine levels.

These results clearly show that nicotine protects tobacco plants in their native habitat, the authors conclude, and that tobacco-chewing insects "prefer low nicotine diets." Removing nicotine from the equation reveals the relentless pressure that plants face from herbivores. Without such defenses, plants would be unceremoniously eliminated posthaste, leaving a world without greenery, not to mention oxygen. But these experiments also demonstrate the unprecedented power of transgenic tools to peel back the obfuscating layers inherent in ecological interactions to reveal the fundamental properties of those interactions. And that scientists working to unravel the tangled web of ecological interactions would do well to take advantage of the longest running experiment on earth-the natural environment.

Steppuhn A, Gase K, Krock B, Halitschke $R$, Baldwin IT (2004) Nicotine's defensive function in nature. DOI: 10.1371/journal. pbio.0020217 
Deconstructing Genetic Contributions to Autoimmunity

in Mouse Models

DOI: 10.1371/journal.pbio.0020220

Given the overwhelming complexity of the immune system, it's no wonder that unraveling the mechanisms responsible for immunological disease has proved so difficult. The factors that trigger autoimmunity-which involves a breakdown in the body's ability to tolerate its own molecules-are not well understood, though animal studies show that genetic predisposition greatly increases risk. And that's where the real challenge begins. For certain diseases, individuals with defects in both copies of a specific gene invariably develop the disease. But more often, diseases with an inherited component result from the complex interplay of a variety of genes, each contributing a small effect that is typically dependent both on the expression of other genes and on both random and environmental factors.

Researchers have increasingly turned to mice to model autoimmunity in humans and have found the same genetic complexity at work, with different strains of lupus-prone mice having different genes predisposing them to autoimmune disease. One model involves targeted disruption of candidate immune system genes to study their role in disease. Gene targeting experiments modify or remove a gene of interest and then watch for corresponding effects on the organism's physiology. Interpretations of results

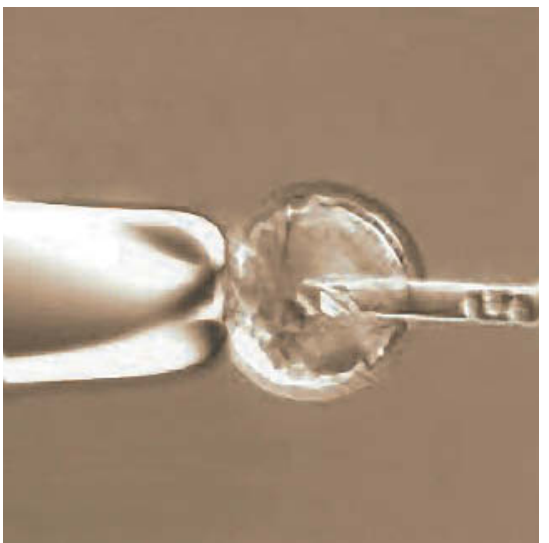

DOI: 10.1371/journal.pbio.0020220.g001

Genetic background can induce autoimmunity in knock-out mice from these experiments have traditionally been predicated on the assumption that the "background," or nontargeted, genes do not contribute to observed physiological changes. Yet in some studies, mice without targeted mutations develop an unexpected susceptibility to the autoimmune disease under study.

To investigate what effects background genes might be having in these mouse models, Marina Botto and colleagues compared the genomes of three hybrid strains of the most commonly used genetic background-the 129 and $\mathrm{C} 57 \mathrm{BL} / 6$ hybrid mice. One of the hybrids, which carries a mutation in both copies of the Apcs gene, was chosen as an example of a gene-targeted model that develops a lupus-like disease, offering an opportunity to examine the relative contributions of the targeted versus

background genes. Apcs is a candidate gene for human systemic lupus erythematosus (SLE), a form of autoimmunity marked by chronic inflammation resulting from a sustained attack on antibodies throughout the body.

Botto and colleagues found that several genomic regions from both the 129 and C57BL/6 mice contributed to autoimmunity, even in the absence of gene-targeted mutations. All of the hybrid strains developed autoimmunity, though disease was more severe in mice with Apcs mutations. Disease outcome in gene-targeted mice, it turns out, can be influenced not just by disease-susceptible (or disease-resistant) gene variants near the targeted gene (in this case, Apcs) but by the random heterogeneity of the hybrid genetic background: multiple combinations of genes in the hybrids can produce the same result.

These results fall in line with mounting evidence that background genes are not silent partners in gene-targeted disease models, but can themselves facilitate expression of the disease. This finding underscores the notion that genes are not solitary, static entities; their expression often depends on context. With genetically complex diseases, having the requisite combination of susceptibility genes does not always lead to disease. Much work remains to be done to identify the triggers that cause the immune system to turn on itself. For more on mouse models and autoimmunity, see the primer by Morel in this issue.

Bygrave AE, Rose KL, Cortes-Hernandez J, Warren J, Rigby RJ, et al. (2004) Spontaneous autoimmunity in 129 and $\mathrm{C57BL} / 6$ mice-Implications for autoimmunity described in genetargeted mice. DOI: 10.1371/journal.pbio.0020243
Identifying Genes Involved in Innate Immunity through RNAi

DOI: 10.1371/journal.pbio.0020249

An organism's ability to sense and respond to potentially harmful pathogens is key to its survival. To fight off disease and infection, organisms must detect pathogens, activate immune cell signaling pathways, and produce molecules able to thwart a pathogenic attack. So fundamental is this need that molecules and protein domains related to innate immunity are evident in organisms as diverse as plants, flies, and humans, highlighting the ancient origins of defense mechanisms. Once detected, the pathogen's presence triggers a cascade of signaling events that generate a rapid response tailored to specific classes of pathogens.

When pathogens attack the Drosophila fruitfly, they elicit a range of defensive reactions in the fly, including the production of antimicrobial proteins, phagocytes (which engulf the pathogen), and toxic metabolites. A microbe sets off one of these responses by interacting with a receptor, triggering a pathway that activates a special class of transcription factors, which in turn activate genes needed to make antimicrobial peptides, say, or toxins. Drosophila attacked by fungi and a certain class of bacteria activate these transcription factors through a pathway (the Toll pathway) that also operates in mammals.

A second pathway is activated when a different class of bacteria attack. While the general steps of this pathway, called the Immune deficiency (Imd) pathway, are known-Dredd-mediated activation of the Relish transcription factor, for example, is central to this antibacterial response-the details and mechanisms remain unclear. Signaling pathways are notoriously complex and the Imd pathway is no different. In the current model, bacterial pathogens stimulate a transmembrane receptor, which activates the Imd protein, which then transmits the signal through intermediary proteins, which ultimately activate Dredd, sending Relish into the nucleus to activate genes required for an immune response.

In this issue of PLoS Biology, Edan Foley and Patrick O'Farrell use a genome-wide approach to characterize the Imd pathway, with an eye toward understanding what regulates the Dredd-Relish interaction. To identify 


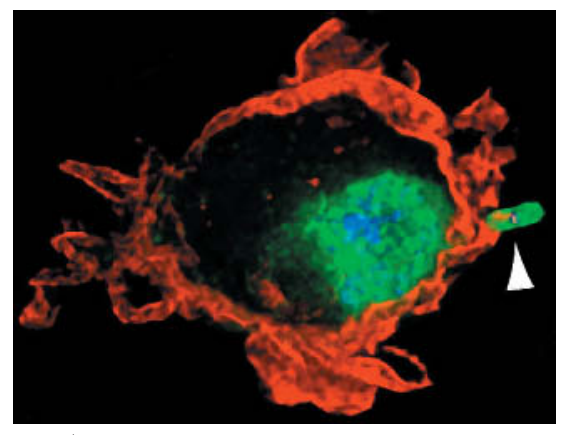

10.1371/journal.pbio.0020249.g001

The NF-kB homolog Relish (green) accumulates in the nucleus of an $\mathbf{S 2}$ cell in contact with E. coli (green rod on cell surface)

pertinent genes and their roles, Foley and O'Farrell took advantage of a technique, called RNA interference (RNAi), that can selectively target and "silence," or inhibit, nearly any gene. After silencing a gene, researchers can then track how a cell responds and infer the gene's function. The technique uses double-stranded RNA (dsRNA) molecules with nucleic acid sequences that match a gene of interest (RNA can bind to DNA through complementary base pairing). The
dsRNAs precipitate a series of steps that ultimately degrade the messenger RNA associated with the gene, preventing messenger RNA translation, thereby silencing the gene.

The authors produced over 7,000 dsRNAs corresponding to most of the Drosophila genes with counterparts in mammals or the worm C. elegans (that is, conserved genes), then developed a cell culture model to analyze the components of the Imd pathway. Since the cells in these cultures respond to the presence of bacterial proteins by generating antimicrobial peptides, they provide a good testbed for identifying genes involved in the pathway. Foley and O'Farrell's RNAi screen identified many molecules involved in signaling, including both signal inhibitors and activators. Among these signaling components, they discovered two new genes: one, which they named sickie, is required to activate Relish; the second, called defense repressor 1 (dnr1), appears to inhibit Dredd activity and thus inhibit the pathway. Based on molecular analysis of these genes-which involved exposing cells to bacterial proteins-the authors propose a model of Imd signaling in which Dredd and the protein produced by $d n r 1$, called Dnr1, operate through a negative feedback loop: Dredd activity appears to promote the accumulation of its own inhibitor, Dnr1. Since suppression of Dnr1 through RNAi can trigger an immune response, the authors explain, it appears that interruption of this feedback loop activates the signaling pathway. Without the inhibitory action of Dnr1, Dredd can activate Relish, which dissociates from Dredd, enters the nucleus, and activates the transcription of antimicrobial genes.

It's likely that the two genes described here play a key role in activating Relish, the authors conclude, and that others identified in this screen will also prove significant. Since many of these components are conserved in mammals, the pathway may likewise operate in humans. Future experiments will be the test of these assumptions, but until then, Foley and O'Farrell have demonstrated the soundness of using RNAi screens on a large scale to dissect the elements of a complex signaling pathway.

Foley E, O'Farrell PH (2004) Functional dissection of an innate immune response by a genome-wide RNAi screen. DOI: 10.1371/ journal.pbio.0020203

\section{A Protein Required for Fruitflies to Dispatch Wasp Parasites \\ DOI: 10.1371/journal.pbio.0020255}

For a three-millimeter invertebrate, the Drosophila fruitfly has a remarkably sophisticated immune system. Granted, it can't customize an immune response by grooming cells to "remember" and target specific pathogens. But it can rally the less specialized tools of innate immunity to fight disease and infection, and in so doing draws on several aspects of blood cell development (called hematopoiesis) - the foundation of the cellular immune response-also found in vertebrates.

In fruitflies, as in vertebrates, hematopoiesis occurs in distinct stages and locations, with nascent cell populations migrating to establish new hematopoietic frontiers. A population of progenitor cells generates all the blood cell types in the organism. These cells arise in two distinct waves and in two distinct locations, with their progeny differentiating into the specialized tissues and organs of the hematopoietic system. In mammals, these organs include the liver and bone marrow, an ongoing source of blood cells after embryogenesis. In fruitflies, the definitive hematopoietic organ is the lymph gland, which churns out three types of blood cells: plasmatocytes and crystal cells, which are also produced by embryonic hematopoietic precursors, and lamellocytes. Plasmatocytes account for up to $95 \%$ of circulating fruitfly blood cells and act much like their mammalian counterpart, the macrophage, engulfing substances deemed foreign and dangerous. Crystal cells account for most of the rest and are involved in melanization reactions, which trigger mechanisms involved in containing and killing invading

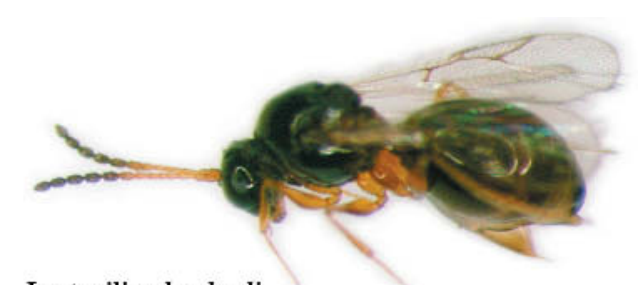

Leptopilina boulardi

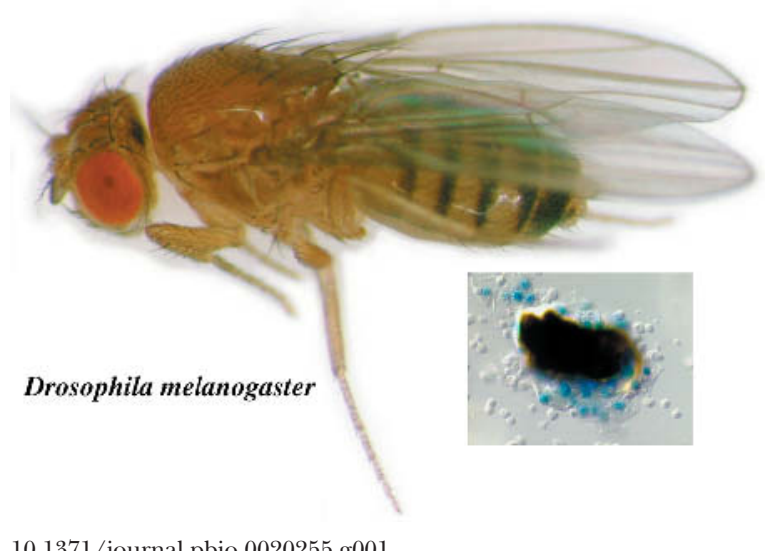

10.1371/journal.pbio.0020255.g001

\section{Cellular immune response to parasitization in Drosophila requires the EBF ortholog Collier}

microbes. Unlike plasmatocytes and crystal cells, lamellocytes appear en masse and only under certain conditions, such as the unwelcome appearance of parasitic wasp eggs. Lamellocytes encapsulate and neutralize the invader. 
Molecular factors involved in determining the fate of hematopoietic cells have been identified for plasmatocytes and crystal cells but not for lamellocytes - until now. In a search for genes that might precipitate lamellocyte differentiation, Marie Meister, Alain Vincent, and colleagues homed in on a protein, called Collier (Col), that is expressed in lymph glands at the end of embryogenesis. Col is quite similar to a mammalian protein, called Early B-cell Factor (EBF), that controls B-cell development in mice. Both proteins are transcription factors, exerting control by initiating gene transcription.

To investigate Col's part in lamellocyte development, the researchers had to get around the fact that mutations that render Col nonfunctional eventually kill the embryo. Using tricks of the genetics trade, Meister and colleagues generated fly larvae that survive loss-of-function mutations in the gene that encodes Col, allowing them to study its role in hematopoiesis.

The mutants had normal amounts of circulating plasmatocytes and crystal cells, but when exposed to parasitic wasp eggs, could not muster the requisite response: lamellocyte production. With no lamellocytes, fly larvae had no means of protection against encroaching wasp eggs, which, uncontested, developed into parasitic larvae. The flies with normal Col levels had no such problem, producing considerable numbers of lamellocytes. In these flies, Col expression was restricted to a lymph region called the posterior signaling center (PSC). Col's influence on lamellocyte fate was strong enough that forcing Col expression in precursor blood cells induced lamellocyte differentiation even in the absence of wasp infestation. Based on these findings, Meister and colleagues propose a model for Col-mediated lamellocyte differentiation in which wasp infestation activates Col-expressing cells in the PSC, which then instructs immature blood cells in the lymph gland to become lamellocytes and dispatch the gathering threat.

Col's role in fruitfly hematopoiesis closely parallels that of its mammalian ortholog in white blood cell development, EBF. Both are required to generate specialized populations of cells in response to a particular immune threat, and both confer an extra line of defense when faced with special circumstances-key features of vertebrate adaptive immunity. Could it be that building blocks of adaptive immunity were already in place some 550 million years ago, when flies and vertebrates parted ways? Researchers will have to investigate the molecular agents of immunity in intervening species to find out.

Crozatier M, Ubeda JM, Vincent A, Meister M (2004) Cellular immune response to parasitization in Drosophila requires the EBF orthologue Collier. DOI: 10.1371/journal.pbio.0020196

\section{The Molecular Biology of Wound Healing \\ DOI: 10.1371/journal.pbio.0020278}

Anyone who's endured their share of childhood scrapes has probably heard some version of the motherly admonishment, "Don't pick that scab, you'll just make it worse!" It turns out, Mom was on to something. Tissue damage in humans triggers a well-characterized response marked by rapid blood clotting and a recruitment of epidermal cells to the injury. When you remove a scab, you're also removing some of the newly regenerated tissues growing underneath, thereby interfering with the healing process.

Many different cell types and proteins have been linked to the repair process, but the complexity of the mammalian wound response has challenged efforts to determine their individual roles. Only the first stepthe signaling cascade that promotes blood clotting -is understood at the molecular level. Since dissecting the wound response pathway at the molecular level requires an organism that lends itself to relatively easy genetic manipulations, Michael Galko and Mark Krasnow of Stanford University turned to the quintessential genetics organism, Drosophila melanogaster, to create

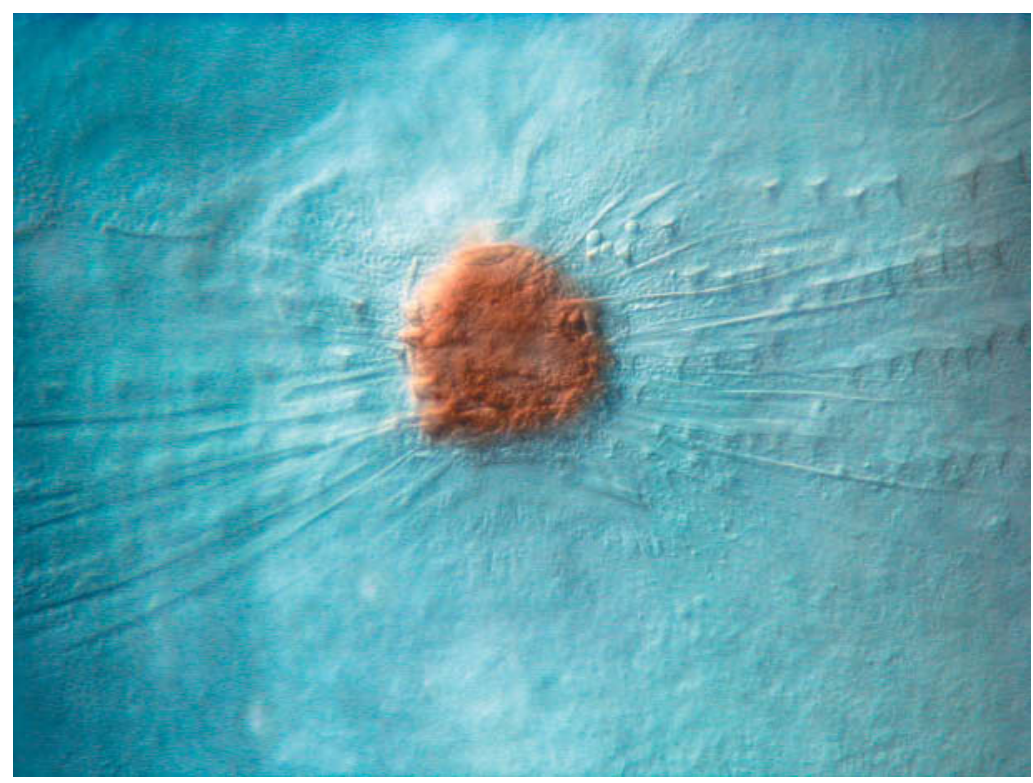

DOI: 10.1371/journal.pbio.0020278.g001

\section{A syncytium forms after wounding the Drosophila epithelium}

a novel system for studying wound healing. After stabbing fruitfly larvae with a needle to create a nonfatal puncture wound, Galko and Krasnow then analyzed significant morphological, cellular, and molecular events at various stages of healing.

Immediately after wounding, the larvae begin to bleed, and about 15 minutes after wounding, the wound darkens and a "plug" of cellular debris forms in the wound. The plug staunches the bleeding and provides a protective layer. Within two hours the plug's outer layer forms a dark, tough scab that presumably serves as an effective barrier to further blood loss. Two or three days later, the healing process is complete.

Galko and Krasnow observed the activity of epidermal cells during healing by labeling their nuclei with a fluorescent protein and staining their membranes. Soon after wounding, cells along the wound margin elongated and aligned themselves toward the wound, then fused together to form a large, multinucleate (multiple nuclei) epidermal cell surrounding the scab. Over the next six hours, these cells also spread along and through the plug, re-establishing a continuous epidermis.

Since this epidermal spreading resembles that seen during a developmental stage of the fruitfly, where the process depends on the JNK signaling pathway, 
the authors investigated JNK signaling to shed light on the genetics and cellular events of healing. Sure enough, they found that the JNK pathway was activated during the peak hours of wound healing. Inhibiting the pathway in fly mutants-the classic approach in fly genetics - had dramatic effects on the wound-healing process. The early stages of healing-including plug and scab formation-weren't affected, but epidermal spreading to regenerate the intact epidermis was either blocked or defective. In contrast, larvae with defects in a gene required for the generation of crystal cells-a type of blood cell implicated in processes linked to scab formation-could not properly form scabs. In these scabless wounds, the JNK pathway was hyperactive, epidermal cells at the wound's margin started to spread to close the wound but often failed, and the wound did not heal. Score one for Mom.

But apparently Mom wasn't totally right: As Galko and Krasnow discovered, a scab isn't always necessary. When the authors pinched larvae without puncturing the overlying cuticle, the wounds did not form scabs. However, they still saw many of the same processes at work around this "pinch" wound that they saw around the puncture wound, and the pinch wounds healed efficiently. This indicates, the authors argue, that the scab functions primarily to provide stability to the wound site and help restore tissue integrity when both the epidermis and overlying cuticle are damaged during wounding.

While the stages of wound healing outlined here occur at a particular time and place, these results suggest that each stage is controlled by distinct genetic programs and signaling pathways triggered by the wound. Since many aspects of the fly wound response resemble those in mammals, it's likely that the molecular components are also shared. That makes identifying the molecular underpinnings of wound healing a high research priority. And thanks to the powerful system presented here, this task should be all the easier.

Galko MJ, Krasnow MA (2004) Cellular and genetic analysis of wound healing in Drosophila larvae. DOI: 10.1371 /journal. pbio.0020239

\section{Retrovirus Integration into the Human Genome}

DOI: 10.1371/journal.pbio.0020281

When gene therapy was introduced nearly fifteen years ago, it was widely hailed as a panacea. Since many diseases have a genetic component, the hope was that gene therapy could replace compromised genes with healthy versions to treat everything from inherited disorders like cystic fibrosis to cancer and HIV. That great promise was quashed when a teenager suffering from a rare hereditary liver disorder died after participating in an experimental gene therapy trial in 1999: four days after being injected with millions of viruses engineered to deliver healthy genes to his liver, Jesse Gelsinger died. It seems the virus, derived from an adenovirus, targeted his immune cells rather than his liver cells, which triggered an immune response against the virus, resulting in massive organ failure.

In another case, two young boys who received gene therapy for the severe immunodeficiency disorder known as "bubble boy disease" developed leukemia-like symptoms 30 months after treatment. In this case, the viral vector inserted itself near a promoter region - a site that initiates gene transcription-of a proto-oncogene, a gene that can initiate cancer. Since viral vectors can integrate at various genomic locations, the safety and effectiveness of gene therapy ultimately depends on being able to predict a virus's particular bias. Comparing retroviral vectors derived from three viruses, including two common gene therapy vectors, Rick Mitchell et al. report 3,127 sites where these viruses typically integrate into the human genome. The different vectors, they found, show different target preferences.

Retroviruses use viral enzymes to copy their own genome, which is stored in an RNA transcript, into DNA. Now recognizable by the host's genome, the virus can integrate into one of the host's chromosomes. In this study, Mitchell et al. studied vectors derived from the human immunodeficiency virus (HIV), avian sarcoma-leukosis virus (ASLV), and murine leukemia virus (MLV). Introducing the viral vectors into human cells, the authors analyzed the gene expression profiles of the cells to determine where vectors integrate into human chromosomes and which, if any, genes they activate. Mitchell et al. then compared the integration sites with the transcription profiles.

Each retrovirus, they discovered, showed distinct preferences for genome integration. HIV vectors tend to integrate into sites of active transcription, favoring chromosomal regions rich in expressed genes. MLV vectors tend to integrate near transcription initiation sites, confirming the results of a previous study, with a weak bias toward active genes. In contrast, the authors report, the ASLV vector "does not favor integration near transcription sites, nor does it strongly favor active genes."

Early efforts to understand how chromosomes may influence where viruses insinuate themselves into a chromosome focused on factors governing accessibility. Viruses are more likely to be integrated into chromosomal regions that are more accessible, which tend to be transcriptionally active sites. But since each of the three viruses studied here routinely targeted different sequences, the authors note, accessibility is probably just one factor. Specific chromosomal proteins, for example, might interact with the viral integration machinery and facilitate integration at nearby sites. Another possibility, the authors propose, is that DNA-binding proteins that bind to specific DNA sequences assist integration of one virus while impeding another.

This could explain why ASLV behaved as it did in the human cells studied here. The virus might have more refined integration preferences during normal infection of chicken cells, the authors note, but its integration machinery can't interact properly with human cells. The leukemia-like effects of the bubble boy gene therapy stemmed from integration of a mammalian retrovirus-the MLV vector-near an oncogene promoter region. Since ASLV tends to avoid both transcription initiation sites and active gene sites, it could be a more promising candidate for human gene therapy. With the draft chicken genome sequence now complete, researchers can investigate whether that proves true. But for now, Mitchell et al. make the case that scientists can gain more control over where viral vectors integrate into the human genome by selecting different retroviral integration systems. Only time will tell whether more control translates into safer gene therapy protocols.

Mitchell RS, Beitzel BF, Schroder SRW, Shinn P, Chen H, et al. (2004) Retroviral DNA integration: ASLV, HIV, and MLV show distinct target site preferences. DOI: 10.1371/journal. pbio.0020234 
Deterministic Tumor Evolution

DOI: 10.1371/journal.pbio.0020284

The essential difference between

cancer cells and normal cells is that cancer cells evolve. Most cancers arise from a single cell through a sequential evolutionary process of mutation and selection. Cancer cells harbor mutations in a number of critical genes that, at various stages during the evolution of the tumor, provide those cells with a selective advantage. Many of the phenotypes, or physical outcomes, conferred by these mutant genes are subverted from a normal cell's repertoire, including proliferation, invasion, migration, loss of differentiation, and loss of apoptosis (programmed cell death); other phenotypes, such as immortalization, are novel.Tumor evolution is thought to adhere to Darwinian principles, with mutations arising randomly within an individual cell, followed by selection for mutant clones with favorable traits.

Support for this idea stems from the observation that end-stage tumors have mutations in a number of genes. But linking mutations in particular genes with defined stages is difficult for most human cancers and especially so for the last and deadliest stage: when cancer disseminates throughout the body during metastasis. It's unclear, for example, whether there are mutations in particular genes or sets of genes that enhance metastasis. That is, do metastatic lesions develop through a continuation of Darwinian evolution, or is metastasis an intrinsic property of the primary tumor, meaning that further genetic evolution is not required? It is also unclear whether there is a "preferred"

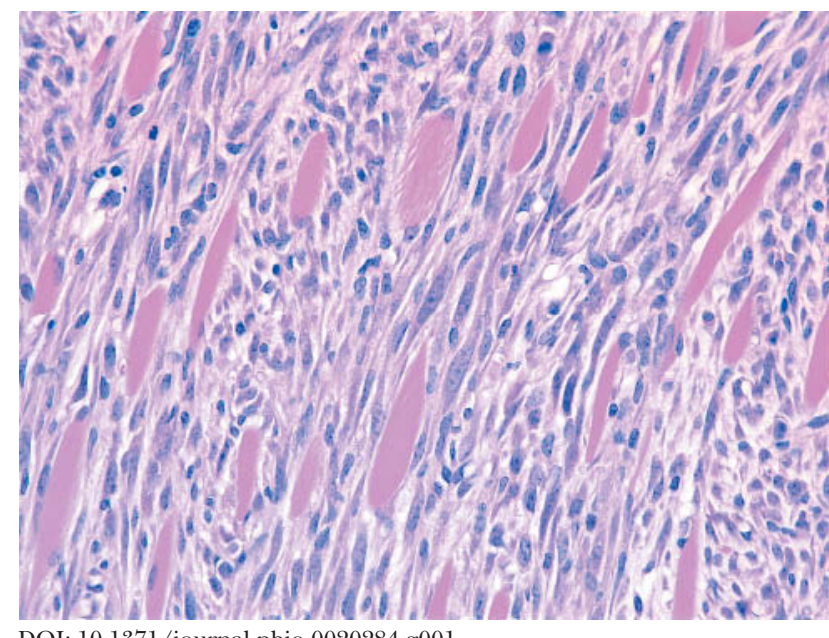

DOI: 10.1371/journal.pbio.0020284.g001

Squamous cell carcinoma invading muscle layer from ARF-null mouse

PLoS Biology | www.plosbiology.org sequence of mutations, such that selective pressure for particular mutations depends on preexisting mutations.

Since the earliest days of research on oncogenes-genes that can cause a cell to become cancerous - it has been known that certain oncogenic mutations cooperate to transform normal cells into cancer cells. For example, an "activating" mutation in the oncogene Ras and the loss of the tumor suppressor $p 53$ cooperate to transform cells.

The paper by Christopher Kemp and his colleagues at the Fred Hutchinson Cancer Research Center sheds light on some of these questions, and many of the issues center around the most notorious oncogene: Ras. Using a well characterized mouse model of squamous cell carcinogenesis, which generates a form of skin cancer, the authors examine both the functional and evolutionary relationships between three cancer genes that play major roles in most human cancers: Ras and the tumor suppressors Arf and p53.

Two seminal early observations set the stage: mutational activation of Ras is the initiating genetic event in this cancer model, while mutation of $p 53$ occurs later, during the benign to malignant transition; and expression of mutant Ras in cells activates $p 53$ via signaling through the protein encoded by Arf.

Kemp et al. confirm that this pathway is active in "autochthonous" tumorswhich grow and develop where they are initiated-by showing that $p 53$ expression in tumors with Ras mutations is dependent on the presence of Arf. Thus, during the early benign stages of tumor growth, Ras activates Arf, which in turn activates $p 53$, thereby inhibiting tumor progression. This provides strong selective pressure in favor of cells with mutations in either Arf or p53, and these mutations are indeed observed as the tumors progress to malignancy. That Arf and p53 function as tumor suppressors was confirmed by demonstrated accelerated tumor progression in mice lacking either Arf or p53.This answers a longstanding question concerning the nature of the signal that activates $p 53$ during autochthonous tumor development: Mutation of Ras not only initiates tumor development but, through its intracellular signaling through $\operatorname{Arf}$ and $p 53$, directly influences the subsequent evolutionary trajectory of the tumors. In this view, secondary evolutionary events are determined by the preexisting genetic lesion, as a result of direct signaling interactions.

The authors go on to show that tumors lacking Arf or $p 53$ show accelerated metastatic dissemination, a phenomenon rarely seen in mouse squamous cell cancer models. Thus both benign and malignant tumors lacking these tumor suppressors are at high risk for metastasis. As Ras is well known to confer many phenotypes required for the metastatic process, it appears that Ras, together with loss of its inhibitors, Arf and p53, may be sufficient to drive this process. More direct evidence that metastasis does or does not require further genetic evolution awaits.

Kelly-Spratt KS, Gurley KE, Yasui Y, Kemp CJ (2004) p19 ${ }^{\text {Arf }}$ suppresses growth, progression, and metastasis of Hras-driven carcinomas through p53-dependent and -independent pathways. DOI: 10.1371/ journal.pbio.0020242

\section{Inhibition of the DNA Damage} Pathway by a Telomere-

\section{Binding Protein}

DOI: 10.1371/journal.pbio.0020270

To maintain the integrity of their genetic content, cells closely monitor the state of their chromosomal DNA. Any break or pairing anomaly in the double helix is perceived as damage that must be repaired. Mechanisms that sense DNA damage enlist the help of cell cycle checkpoint proteins, which stall cell division until the damage has been repaired. The exposed ends of linear chromosomes of eukaryotic cells (cells with nuclei) resemble the double-strand breaks of damaged DNA, which should make them vulnerable to unnecessary manipulations by repair enzymes. But chromosomes have special DNA sequences at their tips, called telomeres, that are coated with telomere-binding proteins that appear to protect ends from the unwanted attentions of repair enzymes. For instance, removing a telomere-specific DNA-binding protein, called TRF2, from telomeres leads to a rapid attack on chromosome tips, 
associated with the activation of the DNA damage pathway. The biochemical basis for TRF2's shielding effects remains obscure.

In this issue of PLoS Biology, Jan Karlseder et al. propose that TRF 2 is a direct inhibitor of an early mediator of the DNA damage signal. Their observations offer new insights into how telomeres resist the inappropriate interventions of the DNA repair machinery.

Ataxia telangiectasia mutated (ATM) kinase is an enzyme that induces the activation of DNA repair enzymes as well as regulators of the cell cycle and apoptosis. Its enzymatic function is triggered by DNA damage sensors. Several observations suggest an antagonism between TRF2 and ATM: ATM is activated in aging cells with shortened telomeres and participates in ushering the cell into replicative senescence. (Cells divide only so many times during their lives before forgoing the process altogether.) In contrast, TRF2 overexpression protects shortened telomeres from decay and delays cell entry into senescence.

Here the authors examine the effect of TRF2 overexpression in cells subjected to radiation-induced DNA damage. This treatment is expected to lead to cell cycle arrest, an outcome mediated for the most part by ATM activation. Irradiated cells that overexpress TRF2, however, continue to enter cell division. Known targets of ATM's enzymatic activity are activated to a lesser degree in these cells, which harbor only about half the amount of active ATM detected in controls. This suggests that TRF2 may interfere with the DNA damage pathway early on, when ATM is activated.

Do these findings reflect a direct interaction between ATM and TRF2? Karlseder et al. suggest they might. Though only a small fraction of TRF2 associates with ATM in normal cells, ATM and TRF2 proteins can form a complex in a test tube. The region of the ATM protein that interacts with TRF2 is the very region required for ATM's activation by DNA damage sensors. The authors propose that TRF2 binds to inactive ATM, and in so doing, prevents ATM's transition to an active state.

The authors integrate their results in an elegant proposal that relates a cell's health to the length of its telomeres. They have previously shown that TRF2 binds another protein called Mre11, a DNA damage sensor known to activate ATM. Thus, by inhibiting ATM, TRF2 may nip in the bud any misguided attempts by Mre11 to "repair" DNA breaks in telomeres. But in aging cells, whose shortened telomeres no longer retain large amounts of TRF2, ATM activation, no longer muffled, eventually allows the onset of senescence. While TRF2 is abundant in normal cells, the authors note that its strict association with telomeres should locally limit its effect on ATM, leaving the DNA damage pathway intact at other chromosomal locations.

Karlseder J, Hoke K, Mirzoeva OK, Bakkenist C, Kastan MB, et al. (2004) The telomeric protein TRF2 binds the ATM kinase and can inhibit the ATM-dependent DNA damage response. DOI: 10.1371/journal.pbio.0020240

\section{Evolution's "Molecular Clock": Not So Dependable After All?}

DOI: 10.1371/journal.pbio.0020287

DNA mutates, and it's a good thing it does. If it didn't, there could only be one kind of life, not the millions there are today, and species could not adapt to new challenges. This is because mutations in genes-the coding portion of DNA - are the raw material for evolution. However, genes make up a surprisingly small fraction of our DNA. If the genome were a cookbook, its 30,000-odd genetic recipes would be scattered among millions of pages of apparently meaningless nonsense.

Mutations affect all DNA, not just the genes, and this provides population geneticists with a veritable toolbox of methods useful, for example, in DNA profiling. Importantly, all these methods rely on the idea of a "molecular clock," the notion that mutations rain down on noncoding DNA like a fine drizzle, so constantly that genetic similarity is a good measure of evolutionary time. Thus, if orangutans diverged from humans twice as long ago as did chimpanzees, on any given piece of DNA we would find twice as many differences between the orangutan sequence and the human sequence as between humans and chimps. The mutations are marking time.

If the molecular clock works, scientists can do wonderful things like estimating how long ago it was that the common ancestor of all humans lived, or when birds evolved from dinosaurs. The clock assumes that mutations occur independently of each other and at a constant rate. By analyzing thousands of noncoding DNA sequences scattered throughout the human genome, Edward Vowles and William Amos

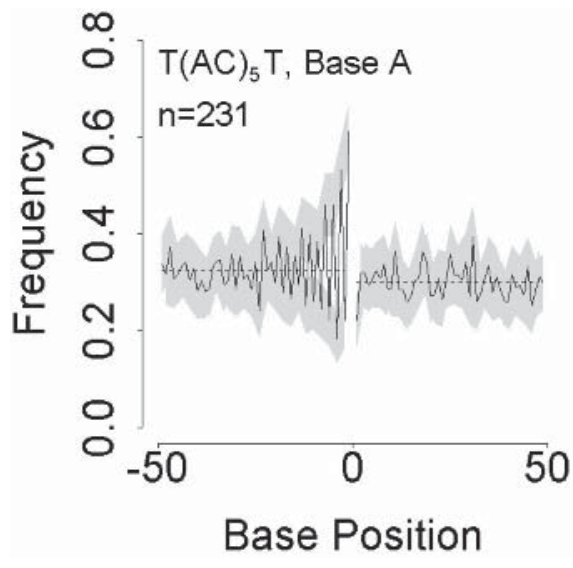

DOI: 10.1371/journal.pbio.0020287.g001

Non-random base frequencies around microsatellites have found that the clock is anything but constant. Instead, a mutation in one spot in the genome affects the chance of getting another mutation nearby.

Not all noncoding DNA is made up of benign tracts of random letters. Some sequences appear to be more difficult to copy than others, and these trouble spots can give rise to alphabetic stuttering. DNA is made up of four component chemical units, called nucleotides, which are often referred to by their initial letters: $A, C, G$, and T. Stuttering occurs when the same pairs or triplets of letters occur together, for example ACACAC. Such regions are called microsatellites, and instead of mutating by swapping one letter for another, as most nucleotides do, these sequences evolve mainly by gaining and losing triplets or pairs like "AC."

In this study, Vowles and Amos used the published sequence of the human genome to track down and compare thousands upon thousands of microsatellites. If the molecular clock ran smoothly, they would expect to find no similarity at all between the DNA sequences surrounding any pair of unrelated microsatellites. To their surprise, they found the complete reverse, with entirely unrelated microsatellites showing widespread and obvious similarities in their flanking DNA. This meant that mutations near microsatellites were not random, but favored certain letters in certain positions. Just as a new shipwreck will attract its own special community of marine life, so microsatellites appear gradually to change the surrounding 
DNA towards a common pattern. The result is convergent evolution, an unusual state of affairs where, as time goes by, DNA sequences become more similar, not less.

As yet, the exact mechanisms remain unclear, though it probably has something to do with how comfortably different combinations of letters sit next to each other. In English, "U" always follows "Q" and "B" never follows "V." Similar rules may apply to DNA, albeit on a much subtler level. For example, if a microsatellite contains alternating $A s$ and $C$, the flanking regions also tend to have As at alternate positions, in phase with the As in the microsatellite. It is as if the DNA prefers the pattern in the microsatellite to extend into the flanking DNA, rather than abruptly stopping at the end of the microsatellite.

These findings suggest that it may be wise to take the notion of a molecular clock at face value. With a perfect clock, two or three identical mutations would be highly unlikely, but we now know that this may be possible near microsatellites. Vowles and Amos estimate that as much as $30 \%$ of the genome may show evidence of convergent evolution, simply because microsatellites are so common. These mutation biases probably exist to a lesser extent in most sequences. Once scientists understand more fully how and where these biases operate, they may be able to estimate more accurately the risk of any given mutation occurring, be it one that causes human disease or makes a virus more virulent. These findings represent yet another windfall from the Human Genome Project, and act as a powerful reminder that unexpected results always lurk around the corner as we delve deeper into the secret world of the genome.

Vowles EJ, Amos W (2004) Evidence for widespread convergent evolution around human microsatellites. DOI: 10.1371/journal.pbio.0020199

\section{Regenerating Lost Muscle: Msx1 to the Rescue \\ DOI: 10.1371/journal.pbio.0020266}

Cell and molecular biologists have a good start at understanding the adult salamander's enviable ability to completely regrow a lost limb or jaw. (Salamanders can even regenerate portions of their eyes and heart.) Happily, mammals share many of the required cellular skills-though in an untapped form. In response to injury, fully differentiated salamander muscle cells can produce less specialized cells that

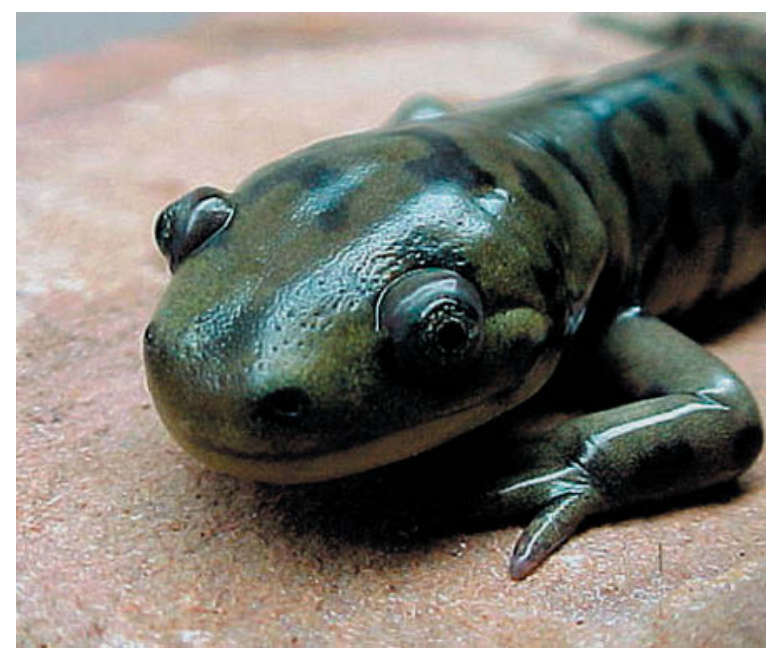

10.1371/journal.pbio.0020266.g001

An adult salamander are capable of multiplying and recreating lost tissue. In this month's PLoS Biology, biochemist Jeremy Brockes and his colleagues at University College London tie this muscle cell de-differentiation to expression of a single gene, $M s \times 1$, a player in limb development and regeneration across species.

The protein encoded by $M s x 1$ appears at the borders of maturing tissues in salamanders, chickens, and mice, and represses differentiation of muscle cells (called myofibers) during development. Msx 1 promotes amphibian limb and tail regeneration, zebrafish fin repair, and regrowth of the tips of digits in mice. Here, Anoop Kumar et al. clarify the gene's role in muscle repair by demonstrating that Msx 1 expression is required for de-differentiation of salamander myofibersmature muscle tissue without the ability to produce new cells-into cells with single nuclei (mononucleate cells) that are able to multiply.

Large, elongated cells, myofibers are distinguished by a striped pattern of actin and myosin, the proteins that produce muscle contractions. They develop from precursor cells, which proliferate and fuse together into multinucleate intermediates called myotubes. As myotubes bulk up on actin and myosin, pushing their many nuclei to the cell periphery, they mature into myofibers. Once differentiated, myofibers are committed to the life of a muscle workhorse. They cannot divide; mature muscle in mammals adds strength and repairs injury by accumulating more actin and myosin, and by fusing single-nucleus "satellite" cells into new or existing myofibers.

Unlike their mammalian counterparts, salamander and newt myofibers respond to injury by splitting into several multinucleated fragments, or by budding off several of their nuclei to create individual cells. These mononucleate progeny multiply and develop to replace lost tissue. Kumar et al. found that more than half of salamander myofibers spontaneously fragment and bud when dissected and placed in cell culture. Prior study has shown that mouse myofibers don't normally behave this way, although cultured mouse myotubes can imitate amphibian myofibers if given a push, in the form of $M s \times 1$. A fraction of mouse myotubes made to express Msx 1 break off mononucleate cells that can be induced to express markers for bone, cartilage, fat, or muscle.

In the salamander cells, Msx 1 RNA and protein appeared in actively fragmenting and budding myofibers, especially in and around their nuclei. When Kumar et al. blocked the translation of Msx 1 mRNA, the myofibers did not generate new cells.

The results confirm Msx 1 as a pivotal regulator of muscle cell re-entry into the cycle of cell division and tissue growth. In this context, it doesn't drive cell division-on the contrary, the authors showed that Msx 1 induced cell splitting without DNA replication. The gene drove committed muscle cells to donate nuclei, creating a pool of new cells to divide, differentiate, and repair damage. Given what it can do for mouse cells in culture, the question remains whether Msx 1 might help awaken a latent capacity for regeneration in living mammals.

Kumar A, Velloso CP, Imokawa Y, Brockes JP (2004) The regenerative plasticity of isolated urodele myofibers and its dependence on Msx1. DOI: 10.1371/ journal.pbio.0020218 


\section{Finding Mutations That Disrupt} Cortical Development

DOI: 10.1371/journal.pbio.0020254

As the presumed "seat of

consciousness," the cerebral cortex

mediates the higher-level cognitive

processing - such as abstract

thought-that humans like to think distinguishes them from other animals.

The cerebral cortex is, in fact, significantly larger in the human brain and has far more "columns" than it does in other mammals, particularly compared to the rat, a traditional model for brain study. Neurons in these cortical columns have similar response properties and form fundamental units of brain processing. It is the larger brain surface area, accommodating a greater number of cortical columns, that gives humans the computational edge.

By studying the genetic and molecular agents of cortex development, scientists hope to understand the nature and extent of cortical cognitive function and identify effective therapies to repair brain injury and disease. Analyses of mutant mice have provided insights into the mechanisms controlling cerebral cortex development, but many details of cortical development remain to be revealed. Identifying individual molecules and genes involved in discrete brain processes is particularly difficult given the complexity of brain structure and function. Geneticists have traditionally linked genes to specific biological pathways by first screening large numbers of individuals of a species for unusual physical traits (phenotypes) and then determining the genetic makeup of these mutants to home in on the faulty gene. This approach, called forward genetics, typically requires large numbers of individuals to find unusual phenotypes and so has traditionally focused on fastbreeding organisms like zebrafish and fruitflies. But zebrafish and fruitflies are unlikely to reveal the secrets of higher consciousness.

Now Andrew Peterson and colleagues have updated the forward genetic screen and added a new resource to the neuroscientist's "brain dissection" toolkit. Their approach, which labels specific populations of neurons with protein "reporters," offers a novel way to find mutations in developing neurons and to identify mutations that interfere with cerebral cortex development. The reporter used here highlights mutations

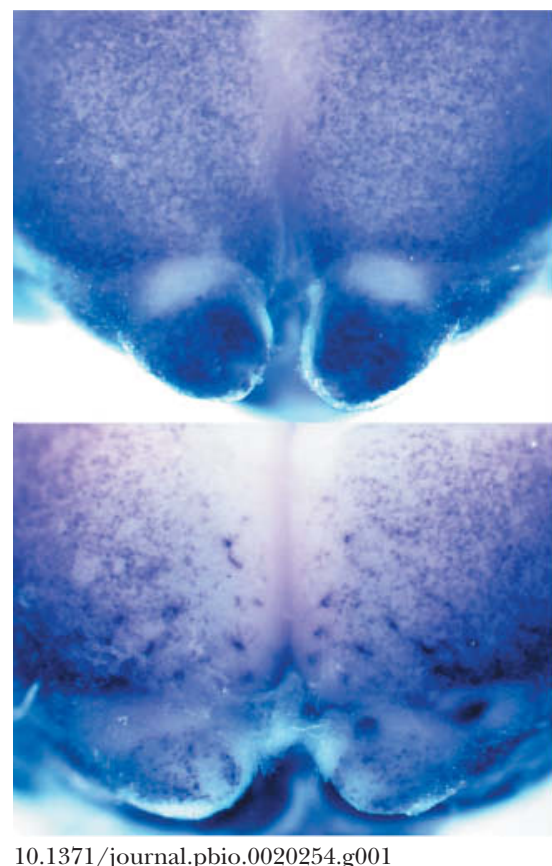

Distribution of GABAergic interneurons in wild-type (top) and mutant (bottom) cortex

that disrupt interneuron migration into the cortex as well as those that affect cortex growth and morphology. (Interneurons are one of the primary types of cortical neurons.)

Like most forward genetic approaches, the researchers started with a genetically well-characterized breed, then used a chemical mutagen to damage the organism's DNA. After two or three rounds of breeding, the researchers looked for cortical-related defects in the developing embryos. In this case, however, the region of the mouse brain that gives rise to developing interneurons was labeled in the original mice. Thus, when the researchers screened for mutants with defects associated with forebrain development and interneuron migration, they could easily find the cells and genes involved.
The screen identified thirteen mutations affecting cortical development and interneuron migration. (Developing neurons travel along the fibers of other brain cells before reaching their ultimate destination in the brain.) Three mutations are variants of genes known to play a role in cortical development; nine mutations were in genes that had not been linked to cortical development before.

The screen described here takes advantage of a chemical mutagen (called ENU) that induces mutations at a single base pair, or nucleotide, in DNA. These mutations tend to have quite selective effects on protein function-by changing the composition of a single domainwhich can provide information on how the protein should normally function and highlight its role in a particular process. The selective nature of ENU, the authors argue, offers new information about how the mutated genes identified here function in cortical development and how these putative roles might be tested.

Altogether, the results suggest that this type of focused screening, so long a resource in fly genetics, can be a powerful tool in mammalian biology as well. That the strategy outlined here could identify novel mutations in a process as complicated as cerebral cortex development suggests that it could do the same for a broad range of biological processes. If the success of other model systems moving in this direction is any indication, this new strategy in the mouse offers researchers a powerful resource for identifying the genetic underpinnings of living systems.

\section{Zarbalis K, May SR, Shen Y, Ekker M, Rubenstein JL, et al. (2004) A focused and efficient genetic screening strategy in the mouse: Identification of mutations that disrupt cortical development. DOI: 10.1371/journal.pbio.0020219}

\section{A Red-Blooded Transcription Factor DOI: 10.1371/journal.pbio.0020282}

Every multicellular organism depends on the coordinated actions of a multitude of cell types, each designed to carry out specific jobs. In vertebrates, for example, the task of ferrying oxygen to organs, tissues, and cells throughout the body is shouldered by red blood cells. These cells must develop early in the embryo to nourish the body and must be maintained at the right levels throughout adulthood to keep the organism healthy.

Specialized red blood cells arise from undifferentiated stem cells in a developmental process called hematopoiesis. All vertebrates have the same basic plan for hematopoiesis during their development, with one group of stem cells producing embryonic blood cells and, later, another group in a different part of the body making adult blood cells. Such developmental programs are tightly orchestrated by suites of transcription factors-proteins that turn genes on and off. 
But which transcription factors act-and how and where they act-is still largely unknown. Scientists and doctors alike want to better understand differentiation in order to grasp not only how it proceeds normally but also how it can go wrong and lead to diseases, such as leukemia, in which white blood cells become cancerous, and aplastic anemia, in which bone marrow stem cells make too few red blood cells.

To discover the genes involved in hematopoiesis, Leonard Zon's group at Children's Hospital, Boston, and their collaborators exposed zebrafish to mutagens and then studied the individuals that developed relevant disorders. In 1996, the group produced three zebrafish lines with embryos lacking fully differentiated red blood cells; since these fish also had especially shimmery tails, the lines were named moonshine.

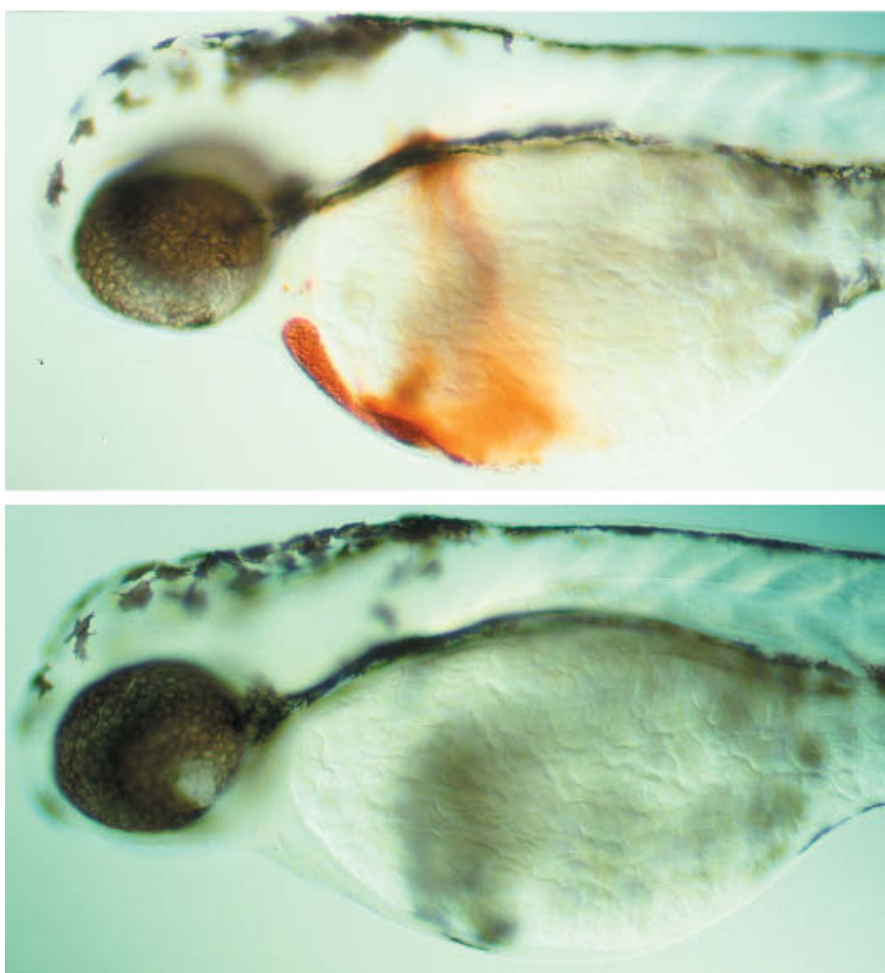

DOI: 10.1371/journal.pbio.0020282.g001

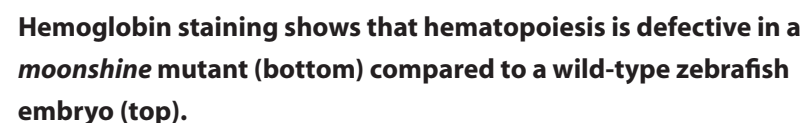
embryo (top).

was unable to keep the blood cells alive. These blood cells underwent apoptosis, or programmed cell death, leaving the fish unable to make red blood cells, and they died at about two weeks old.

The researchers found that the protein encoded by the mon gene is most similar to the human and mouse Tif1-gamma, one of a family of proteins known to link DNAbinding proteins with other factors that activate or repress gene activity. Using a DNA analysis technique in mouse cell cultures, the researchers found Tif1-gamma in nuclear bodies, multi-protein complexes in cell nuclei that help regulate gene expression. But the Tif1-gamma nuclear bodies didn't fit into any known class of such complexes, so it's an open question how Tif1-gamma acts and what other proteins help regulate it. Finding a transcription factor such as Tif1-gamma involved in early cell specialization opens a door to a suite of studies on the targets of the transcription factor, as well as other genes that act along with mon to affect red blood cell development.

Ransom DG, Bahary N, Niss K, Traver D, Burns C, et al. (2004) The zebrafish moonshine gene encodes transcriptional intermediary factor $1 \gamma$, an essential regulator of hematopoiesis. DOI: 10.1371/journal.pbio.0020237
Yeast Use Dual Gain Controls to Amplify Protein Processing DOI: 10.1371/journal.pbio.0020269

Machinery within the endoplasmic reticulum (ER) of eukaryotic cells modifies, folds, and assembles proteins as needed to suit their functions at or past the cell membrane. When this system is hampered or overtaxed, a buildup of unfolded or misfolded proteins within the ER triggers the "unfolded protein response," which alerts the nucleus to boost production of protein-processing machinery that helps proteins fold. This system for adjusting manufacturing capacity is similar in organisms from yeast to human. If the unfolded protein response cannot be turned on when needed, cells die. Prior study suggested that, in yeast cells, the response to unfolded protein buildup is binary: either off or on. In this month's PLOS Biology, biochemist Peter Walter and his colleagues from the University of California at San Francisco demonstrate two new signaling mechanisms that appear to give the yeast unfolded protein response the means for amplitude adjustment.

In yeast, a transcription regulator called Hac $1 \mathrm{p}$ activates the genes required for the unfolded protein response. A cytoplasmic pool of $\mathrm{HAC1}$ messenger RNA waits in readiness for ER emergencies, each molecule locked against translation into protein by intronic RNA sequences that interrupt mRNA translation. Accumulation of unfolded or misfolded proteins in the ER releases this translational block, triggering production of $\mathrm{Hac} 1 \mathrm{p}$ and activating the unfolded protein response. Previously, this binary HAC1 signal

\section{HAC1}

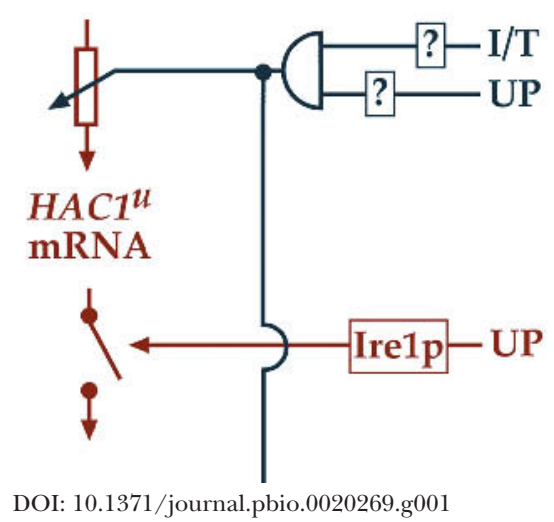

Wiring the unfolded protein response 


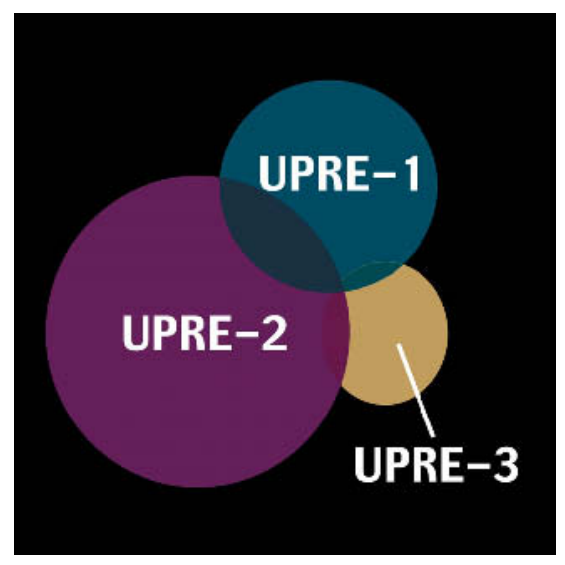

DOI: 10.1371/journal.pbio.0020269.g002

\section{Control elements of UPR target genes}

was the only known regulator of the unfolded protein response in yeast. In two complementary papers, Walter and colleagues now present evidence that the repertoire includes new factors and regulators that amplify the unfolded protein response under conditions of ER stress.

Leber et al. stressed yeast cells by exposing them to substances that cause protein misfolding or buildup in the ER. In response, the cells ratcheted up transcription levels of $H A C 1$ severalfold. Primed with high levels of $H A C 1$ mRNA, the cells were ready to produce a bumper crop of Hac1 $\mathrm{p}$ and to induce a supercharged unfolded protein response. In the accompanying paper by Patil et al., the authors show that Hac1p is not working alone. A second regulator of transcription called Gcn4p is required to activate most of the genes associated with the unfolded protein response. The regulatory elements of these genes now appear far more diverse than previously appreciated. The authors propose that cells adjust the levels of Hac1p and Gcn4p to drive a continuum of transcriptional programs equipped to deal with incoming challenges. Together, these two papers demonstrate that the control of the unfolded protein response is far from a simple on/off mechanism, but exhibits complex fine-tuning through a network of signaling pathways that interpret and respond to the cell's needs.

Patil C, Li H, Walter P (2004) Gcn4p and novel upstream activating sequences regulate targets of the unfolded protein response. DOI: 10.1371/journal. pbio.0020246

Leber JH, Bernales S, Walter P (2004) IRE1independent gain control of the unfolded protein response. DOI: 10.1371 /journal. pbio.0020235
A New Role for a Synaptotagmin

Protein in Calcium-Dependent

\section{Exocytosis}

DOI: 10.1371/journal.pbio.0020253

The hardest working molecules in cell biology, proteins abound in a dazzling variety of shapes and sizes to carry out an equally impressive array of tasks. Many proteins function within the cell, while others get shipped out to locations both near and far. Specialized organelles within the cell package the traveling proteins into cargo containers called vesicles. Vesicles move through a highly regulated transportation system until they reach their ultimate destination, either inside or outside the cell, and release their cargo. When vesicles fuse to the plasma membrane and release their cargo outside the cell the process is called exocytosis. Exocytosis allows macromolecules to leave the cell without compromising the structural integrity of its membrane.

"Professional" secretory cells specialize in producing copious quantities of their protein product and sending vesicles packed with their customized issue to the plasma membrane. Once there, the vesicles wait for a signal to fuse with the membrane. The signal most often comes in the form of a transient and localized increase in calcium ion levels.

Over a decade ago, researchers discovered that calcium-triggered exocytosis also occurs in "nonprofessional" secretory cells. In these cells, the process was thought to be important for healing ruptured plasma membrane, though the identity of the vesicles responsible remained unknown. It has been suggested that these vesicles are lysosomes, enzyme-filled organelles that break down waste and extracellular debris ingested by the cells. Sanford Simon, with his colleagues Jyoti Jaiswal and Norma Andrews, previously confirmed that calcium does specifically trigger exocytosis of lysosomes in the "nonprofessional" secretory cells. Calcium-triggered exocytosis is thought to require the services of a family of proteins called synaptotagmins. But the fifteen members of the synaptotagmin family diverge from this job description in various ways, calling its role into question.

Synaptotagmin VII (Syt VII)—-the synaptotagmin member expressed on the lysosomes-is present in most tissues in organisms ranging from worms to humans. This protein functions in processes requiring lysosomal exocytosis and during invasion by trypanosome parasites, such as the one that causes Chagas disease. In this issue of PLoS Biology, Jaiswal, Simon, and colleagues investigate the molecular mechanisms underlying calcium-triggered lysosomal exocytosis, focusing on the role of Syt VII.

The researchers took cells from two lines of mice: one lacked the functional Syt VII protein and the other produced normal levels of Syt VII. First they labeled the surface and interior cavity of lysosomes in these cells with fluorescent tags and triggered an increase in cells' calcium level; then they watched the behavior of single lysosomes releasing their contents in real time.

Most of the lysosomes from normal cells released only a portion of their contents and had very small fusion pores that remained open to the outside of the cell for only a short time. Interestingly, while proteins in secretory vesicle membranes typically diffuse into the plasma membrane during exocytosis, proteins in the lysosomal membrane stayed near the site of fusion.

In the cells from Syt VII-deficient mice, Simon and colleagues discovered, to their surprise, that this protein isn't necessary for calcium-triggering of lysosomal exocytosis. Calcium-triggered exocytosis not only occurred in these cells, it happened more rapidly than in normal cells. Plus most of these deficient lysosomes fused completely and their membrane proteins fully diffused into the plasma membrane. Simon and colleagues argue that these results show that Syt VII restricts rather than facilitates lysosomal exocytosis. It does so by limiting the formation and size of fusion pores and by preventing lysosomal membrane proteins from integrating into the plasma membrane.

Jaiswal JK, Chakrabarti S, Andrews NW, Simon SM (2004) Synaptotagmin VII restricts fusion pore expansion during lysosomal exocytosis. DOI: 10.1371/journal.pbio.0020233 


\section{How the Brain Retrieves Forgotten Memories \\ DOI: 10.1371/journal.pbio.0020283}

In the 2002 thriller Memento, the protagonist, Lenny, is plagued by a crippling neurological disorder that renders him incapable of storing memories for longer than fifteen minutes. He wants nothing more than to avenge the murder of his wife, but as another character in the film tells him, "Even if you get revenge, you're not going to remember it. You're not even going to know it happened." Lenny has anterograde amnesia, a condition typically caused by stroke or other illness. (In the film, it's caused by a blow to the head.) Most forms of amnesia-including the more common retrograde amnesia, which involves the loss of long-term memory-are caused by some type of brain injuryparticularly to the hippocampus.

Retrograde amnesia can arise from brain damage that interferes with memory storage, retrieval, or consolidation. And it is by studying retrograde amnesia that scientists have developed theories explaining where the brain stores memory traces and how it consolidates them into long-term memories. What ultimately causes amnesia-a failure to store memories or a failure to retrieve them-is not clear.

A major challenge in resolving this question experimentally is being able to determine whether an animal has truly recovered a lost memory or has simply re-learned the task at hand. By refining a classic behavioral neuroscience experiment to test spatial learning and memory, Livia de Hoz, Stephen Martin, and Richard Morris have developed a novel protocol that distinguishes retrieval from new learning. The experiment, developed by Morris over fifteen years ago, takes advantage of the fact that rats are good swimmers but prefer life out of water. Rats are placed in a pool and trained to remember the location of an escape platform, hidden just under the surface. (The rats can't see it because the water is cloudy.)

Here, de Hoz et al. trained rats to a particular platform location-their escape route-then gave them hippocampal lesions. One group received "sham" lesions, another partial lesions, and a third complete lesions. Partial lesions, the authors explain, should damage only a subset of the stored memory traces and thus weaken the rats' memory rather than completely disrupting it.

The rats' postoperative memory was tested by first placing them in the pool with the platform hidden: as expected, the lesioned rats couldn't remember where it was. After a minute, the platform was raised above the water, to remind them that it existed and that they could escape by climbing onto it. The platform was revealed in the original location for half the animals and in a novel location-which could be construed as misleading information if you're a rat-for the other half. If raising the platform was facilitating re-learning, then animals capable of learning would look for the platform in the new location. But if raising the platform functioned as a reminder, then animals should gravitate toward the place they were trained to, regardless of whether the platform reminder was in the original location or a new one. And that's what happened to the rats with partial lesions.

De Hoz et al. then repeated the experiments in a new environment. If the rats managed to escape in this situation, the authors explain, then the reminder treatment could be causing new learning rather than triggering recall of the original training experience. Not surprisingly, the rats with total lesions failed to learn in this new environment (or to be reminded in the previous experiment), while the control rats adapted to the new location. The group with partial lesions failed to learn.

Since partially lesioned rats responded to the reminder treatment by recalling the original platform location, their initial memory failure couldn't have resulted from a storage failure. And since they did not realize they could escape from the new

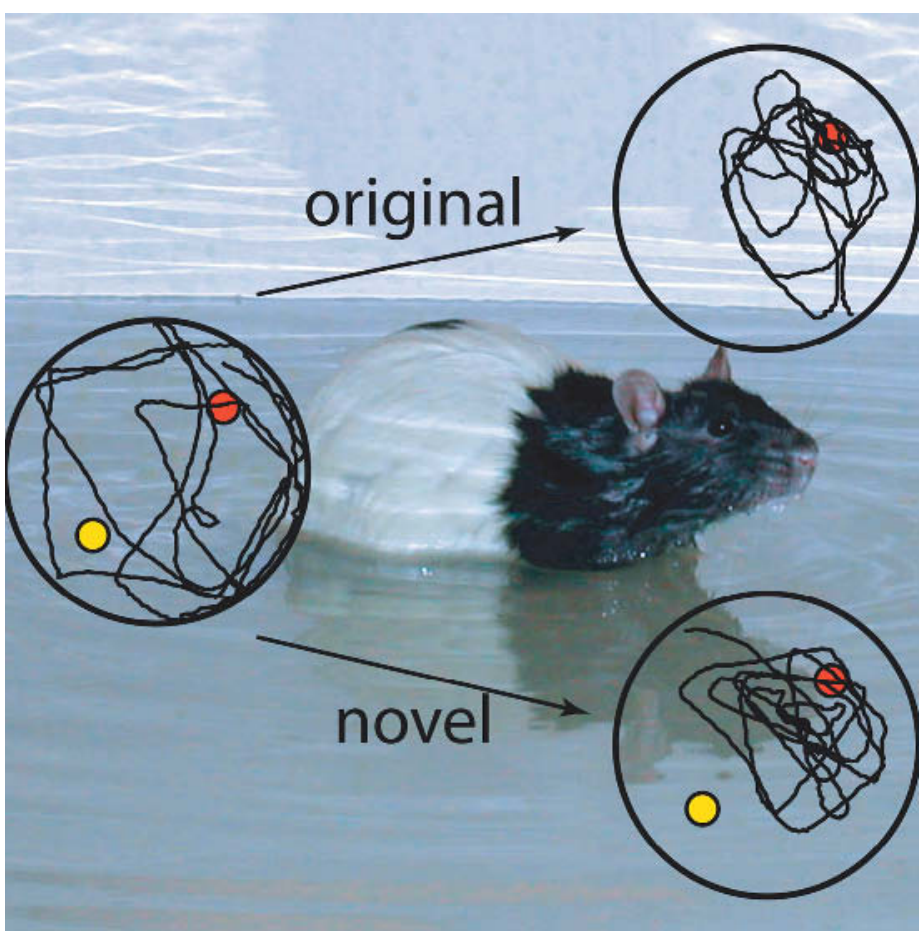

DOI: 10.1371/journal.pbio.0020283.g001

Rats given partial hippocampal lesions after training in a watermaze are initially unable to remember the location of the escape platform.

location, the reminder didn't induce new learning. Among the many questions these results raise is what role the hippocampus plays in memory storage and retrieval. It could be, as the authors propose, that essential components of spatial memory traces are either stored in the hippocampus or reactivated there, since only the partially lesioned rats responded to reminders. Whether that proves true, de Hoz et al. have contributed a much needed resource for investigating the neural basis of memory loss.

de Hoz L, Martin SJ, Morris RGM (2004) Forgetting, reminding, and remembering: The retrieval of lost spatial memory. DOI: 10.1371/ journal.pbio.0020225 